\title{
Determinación del Desgaste por Erosión en Materiales Recubiertos
}

\author{
José L. Caballero(1), Willian A. Aperador(1) y Arnoldo E. Delgado(2) \\ (1) Grupo de Investigación Volta, Facultad de Ingeniería, Universidad Militar Nueva Granada, Carrera 11 No. \\ 101-80, Bogotá-Colombia (e-mail: caballerojl22@gmail.com; g.ing.materiales@gmail.com). \\ (2) Grupo de Investigación Energía Alternativa, Facultad de Ingeniería, Universidad Militar Nueva Granada, \\ Carrera 11 No.101-80, Bogotá-Colombia (e-mail: arnoldo.delgado@unimilitar.edu.co).
}

Recibido Jul. 21, 2014; Aceptado Oct. 6, 2014; Versión final recibida Oct. 20, 2014

\begin{abstract}
Resumen
En el presente artículo se determina la tasa de erosión de recubiertos cerámicos, mediante el uso de una aplicación de software que involucra parámetros relacionados con el proceso de desgaste erosivo. La aplicación fue realizada en SharpDevelop 4.2, un entorno de desarrollo integrado libre para lenguaje C\#. Adicionalmente el programa hace uso de las librerías de código abierto, MySql para hacer gestión de una base de datos que contiene los parámetros de erosión y ZedGraph para graficar los datos. Para contrastar lo obtenido de forma experimental con lo calculado por el software, se estudió la erosión en capas de nitruro de titanio (TiN) y nitruro de cromo ( $\mathrm{CrN}$ ) depositadas sobre sustratos de acero AISI 4140 . Se determinó que la tasa de erosión adquiere su valor máximo cuando el ángulo de incidencia es $90^{\circ}$ y que los valores de desgaste experimentales y calculados correlacionan de forma aceptable.
\end{abstract}

Palabras clave: desgaste por erosión, tasa de desgaste, recubrimientos cerámicos, partícula abrasiva, SharpDevelop

\section{Determining Wear by Erosion in Coated Materials}

\begin{abstract}
In this paper the erosion rate of ceramic coating using a software application which includes parameters related to the erosive wear process is determined. The application was done using SharpDevelop 4.2, a free and open integrated development environment in C\# language. Moreover, the program makes use of the open source libraries MySql to manage the data containing the erosion parameters and ZedGraph to plot the data. Erosion of layers of titanium nitride (TiN) and chromium nitride (CrN) deposited on AISI 4140 steel substrates was experimentally studied and results compared with the software results. Results show that the erosion rate reaches its maximum value when the angle of incidence is $90^{\circ}$ and that experimental and calculated values of erosion rate are well correlated.
\end{abstract}

Keywords: wear rate, ceramic coatings, abrasive particles, SharpDevelop 


\section{INTRODUCCIÓN}

El estudio del desgaste y la fricción, constituyen uno de los últimos aspectos de la mecánica clásica que aún presenta interrogantes abiertos importantes. Bajo la óptica de la ingeniería, su importancia está en aumento, en función de las cargas impuestas a los componentes mecánicos, la disminución constante de las tolerancias permitidas en los procesos de fabricación, así como las crecientes restricciones en cuanto a la emisión de contaminantes, también colocan desafíos a la relativa nueva ciencia de la tribología (Aperador et al., 2010; Caicedo et al., 2012; Aperador et al., 2011a).

El desgaste por erosión, entendido como el daño provocado por el impacto de partículas duras presentes en un fluido, afecta equipos como turbinas, bombas, tuberías y otros dispositivos que transportan fluidos y los cuales pueden estar sometidos a cambios bruscos de presión, provocando efectos como erosión de superficies sólidas, vibraciones, ruidos excesivos, disminución de eficiencia y otros, lo que finalmente se traduce en enormes gastos por reparo o reposición de este tipo de equipos (Aperador et al., 2011a; Aperador et al., 2011; Holmberg, Matthews y Ronkainen, 1998). La erosión (partículas al azar) es una forma de desgaste por impacto que puede ocurrir por chorros y flujos de partículas sólidas pequeñas transportadas por un fluido, en general aire o agua, o también por gotas líquidas. La percusión ocurre por impactos repetidos de cuerpos sólidos de tamaño mayor. (Gee et al., 2014)

Actualmente los nuevos materiales se han convertido en el atractivo de múltiples aplicaciones a nivel industrial. Dentro de estos nuevos materiales encontramos los recubrimientos duros, éstos han sido desarrollados con el fin de aumentar la vida útil de algunos elementos industriales, minimizando así los tiempos de producción, costos e interrupciones por mantenimiento. Entre las técnicas más empleadas para la obtención de dichos recubrimientos está la deposición física fase vapor (PVD), en donde el material que formará el recubrimiento se evapora y posteriormente condensa en forma de una capa sobre el sustrato (Junhua, Geyang y Mingyuan, 2000; Yashar y Sproul, 1999; Caicedo et al., 2010; Nieto et al., 2009). El desgaste es un problema al que se ve enfrentado algunos elementos útiles en la industria del transporte de fluidos, como partes de motobombas, en algunos casos estos fluidos son sustancias agresivas que además contienen partículas en suspensión, que pueden ser causantes de fenómenos de deterioro como lo es la erosión. (Aperador et al. 2014)

Los recubrimientos duros se encuentran aplicados a distintas ramas de la industria tales como la automotriz, aeronáutica, transporte de fluidos, minería y la industria petrolera. Mediante la aplicación de recubrimientos duros a partes útiles a la industria, es posible incrementar su dureza, disminuir el coeficiente de fricción, aumentar la resistencia al desgaste y a la fatiga, con lo que se logra un incremento en la vida útil de dicha parte que puede significar un aumento en la producción de una empresa (Chu y Barnett, 1995; Barshilia et al., 2007; Barshilia et al., 2009; Jehn, 2000; Bautista, Aperador y España, 2011; Caicedo et al., 2011). En este trabajo se desarrolla una herramienta sistematizada que permite calcular la tasa de desgaste de materiales metálicos recubiertos mediante monocapas de TiN y $\mathrm{CrN}$, depositados por medio de la técnica PVD sobre un substrato de acero 4140, se realizó la comparación entre lo obtenido de forma experimental y lo hallado en la simulación, generando una correlación adecuada.

\section{TASA DE EROSIÓN}

La tasa de erosión de una superficie cerámica expuesta a un flujo de lodo abrasivo se puede expresar como el producto de la tasa de erosión característica $(E R)_{c}$ por la función adimensional de tasa local de erosión $E(\theta, \ldots) \approx \bar{E}_{\text {lim }}$, suponiendo que la población de partículas en el lodo es tan grande que todas fluyen con una trayectoria cercana a una línea recta, a una velocidad aproximada a la velocidad de la corriente principal $V_{p} \approx U y$ a un ángulo local de incidencia $\theta_{i} \approx \theta$ (Rosner et al., 1994; Rosner, Tandon y Labuwaky, 1995).

$E R=(E R)_{c} \cdot E(\theta, \ldots)$

La tasa de erosión característica se da en un ambiente donde todas las partículas abrasivas de la corriente principal poseen un tamaño promedio e impactan el objetivo cerámicocon una incidencia normal y una velocidad disminuida. (Khalil y Rosner, 1996)

$(E R)_{c} \equiv \varepsilon_{p}\left(U, 0, \bar{v}_{p, \infty}\right) \cdot\left(\bar{v}_{p} N_{p} U\right)_{\infty}$

Donde $\varepsilon_{p}$ es el rendimiento especifico a la erosión evaluado a la velocidad de la corriente principal $U$, a un ángulo de incidencia normal un volumen de partícula abrasiva $\bar{v}_{p \cdot \infty}, \mathrm{y}\left(\bar{v}_{p} N_{p}\right)_{\infty}$ es la fracción de volumen de la corriente principal $\phi_{p . \infty}$. El volumen promedio de material cerámico removido por unidad de volumen de las partículas abrasivas impactadas puede calcularse de la siguiente manera: (Khalil y Rosner, 1996) 
$\varepsilon_{p}\left(U, 0, \bar{v}_{p . \infty}\right)=\varepsilon_{p . r e f .}\left(V_{p} / V_{\text {p.ref. }}\right)^{n} \cdot \cos \left(\theta_{i}\right)^{m} \cdot\left(v_{p} / v_{p . r e f .}\right)^{l}$

$\varepsilon_{p}\left(U, 0, \bar{d}_{p . \infty}\right)=\varepsilon_{\text {p.ref. }}\left(V_{p} / V_{p . r e f .}\right)^{n} \cdot \cos \left(\theta_{i}\right)^{m} \cdot\left(d_{p} / d_{\text {p.ref. }}\right)^{3 \times l}$

El $\varepsilon_{p . r e f}$. es el valor de $\varepsilon_{p}$ evaluado a una velocidad de impacto de la partícula $V_{p}=100 \mathrm{~ms}^{-1}$, a un ángulo de incidencia $\theta_{i}=0^{\circ}$ y un volumen de partícula $v_{p}$ correspondiente a un diámetro de partícula de $d_{p}=100 \mu m$ debido a que $v_{p}=(\pi / 6) \cdot d_{p}^{3}$. Los parámetros de erosión $\mathrm{I}, \mathrm{m}, \mathrm{n}$ son exponentes adimensionales que describen la sensibilidad del rendimiento a la erosión respecto al tamaño de la partícula, al ángulo de incidencia y a la velocidad de incidencia de la partícula respectivamente. (Bitter, 1963; Crowley, 1969; Finnie, 1960; Keairns, Yang y Vaux 1979)

Los valores de los parámetros de erosión $\varepsilon_{\text {p.ref. }} l, m, n$ se encuentran en la tabla 1 , en la cual se resumen los resultados experimentales del rendimiento a la erosión de una variedad de recubrimientos cerámicos/partículas abrasivas para una temperatura cercana a 300 K. (Gulden, 1979; Wang, She y Ma 1995; Liebhard, 1994; Head y Harr 1994; Wiederhorn y Roberts 1976)

Tabla 1: Combinaciones de recubrimiento cerámico y partícula abrasiva. (Khalil y Rosner 1996)

\begin{tabular}{|c|c|c|c|c|c|c|c|}
\hline No & Material objetivo / Tipo de partícula & $\begin{array}{c}\text { Densidad } \\
{\left[\mathrm{g} / \mathrm{cm}^{3}\right]}\end{array}$ & 1 & $\mathrm{~m}$ & $\mathrm{n}$ & Epref & Fuente \\
\hline 1 & Castable A / Dead burned dolomite & 3 & 1.3 & 1.3 & 3 & $9.6 \mathrm{E}-4$ & Keairns et al (1979) \\
\hline 2 & Castable C / Dead burned dolomite & 3 & 1.2 & 1.1 & 2.5 & $7.2 \mathrm{E}-5$ & Keairns et al (1979) \\
\hline 3 & Castable F / Dead burned dolomite & 3 & 1 & 1 & 2.9 & $7.8 \mathrm{E}-5$ & Keairns et al (1979) \\
\hline 4 & Vidrio / Silica Flour & 2.65 & 1.2 & 2.5 & 2.3 & $7.2 \mathrm{E}-1$ & Head y Harr (1970) \\
\hline 5 & Vidrio / Crystolon & 3.17 & 1.2 & 1.8 & 2.3 & $2.3 \mathrm{E}-1$ & Head y Harr (1970) \\
\hline 6 & Vidrio / Alundum & 4 & 1.2 & 1.8 & 2.3 & $2.4 \mathrm{E}-1$ & Head y Harr (1970) \\
\hline 7 & Vidrio / Perlas de cristal & 2.47 & 1.2 & 6.3 & 2.3 & $5.6 \mathrm{E}-2$ & Head y Harr (1970) \\
\hline 8 & Refractario de alta pureza / Carburo de Silicio & 3.17 & 1.2 & 0.6 & 2.8 & $7.5 \mathrm{E}-7$ & $\begin{array}{l}\text { Wiederhorn y } \\
\text { Roberts (1976) }\end{array}$ \\
\hline 9 & Hormigón refractario aislante / Arena & 2.4 & 1.2 & 0.5 & 2.3 & $1.5 \mathrm{E}-3$ & Crowley (1969) \\
\hline 10 & Hormigón refractario aislante / Blasting grit & 2.65 & 1.2 & 1.4 & 2.3 & $1.4 \mathrm{E}-5$ & Crowley (1969) \\
\hline 11 & $\begin{array}{l}\text { Hormigón refractario resistente a la abrasión C } \\
\text { / Blasting grit }\end{array}$ & 2.65 & 1.2 & 1.6 & 2.3 & $1.6 \mathrm{E}-7$ & Crowley (1969) \\
\hline 12 & $\begin{array}{l}\text { Hormigón refractario resistente a la abrasión C } \\
\text { / Arena }\end{array}$ & 2.4 & 1.2 & 4.4 & 2.3 & $8.9 \mathrm{E}-6$ & Crowley (1969) \\
\hline 13 & $\begin{array}{l}\text { Hormigón refractario de alta resistencia / } \\
\text { Blasting grit }\end{array}$ & 2.65 & 1.2 & 3.6 & 2.3 & $2 \mathrm{E}-6$ & Crowley (1969) \\
\hline 14 & Hormigón refractario de alta resistencia / Arena & 2.4 & 1.2 & 3 & 2.3 & $1.1 \mathrm{E}-3$ & Crowley (1969) \\
\hline 15 & Vidrio recocido / Hierro fundido & 7.8 & 1.2 & 2.5 & 2.3 & $1.4 \mathrm{E}-4$ & Finnie (1960) \\
\hline 16 & Vidrio recocido / Hierro fundido & 7.8 & 1.2 & 2.7 & 2.3 & $1.1 \mathrm{E}-5$ & Finnie (1960) \\
\hline 17 & Vidrio recocido / Hierro fundido (2) & 7.8 & 1.2 & 3.5 & 2.3 & $2.5 \mathrm{E}-4$ & Bitter (1963) \\
\hline 18 & $\begin{array}{l}80 \% \text { Alumina Phos. Bonded castable / Carburo } \\
\text { de silicio }\end{array}$ & 3.22 & 1.2 & 0 & 2.3 & $9.8 \mathrm{E}-5$ & Liebhard (1994) \\
\hline 19 & $\begin{array}{l}\text { Nitruro de silicio bonded SiC brick / Carburo de } \\
\text { silicio }\end{array}$ & 3.22 & 1.2 & 0 & 2.3 & $1.2 \mathrm{E}-4$ & Liebhard (1994) \\
\hline 20 & $\begin{array}{l}\text { Cemento modeable ultra bajo / Carburo de } \\
\text { silicio }\end{array}$ & 3.22 & 1.2 & 0 & 2.3 & $1.2 \mathrm{E}-4$ & Liebhard (1994) \\
\hline 21 & $\begin{array}{l}60 \% \text { Ladrillo de alumina (mullite based) / } \\
\text { Carburo de silicio }\end{array}$ & 3.22 & 1.2 & 0 & 2.3 & $2.0 \mathrm{E}-4$ & Liebhard (1994) \\
\hline 22 & $\begin{array}{l}\text { Phos. bonded plastic, grano fino / Carburo de } \\
\text { silicio }\end{array}$ & 3.22 & 1.2 & 0 & 2.3 & $2.2 \mathrm{E}-4$ & Liebhard (1994) \\
\hline 23 & $\begin{array}{l}\text { Phos. bonded plastic, grano regular / Carburo } \\
\text { de silicio }\end{array}$ & 3.22 & 1.2 & 0 & 2.3 & $2.4 \mathrm{E}-4$ & Liebhard (1994) \\
\hline 24 & Cemento moldeable bajo / Carburo de silicio & 3.22 & 1.2 & 0 & 2.3 & $2.5 \mathrm{E}-4$ & Liebhard (1994) \\
\hline
\end{tabular}


Tabla 1 (continuación)

\begin{tabular}{|c|c|c|c|c|c|c|c|}
\hline No & Material objetivo / Tipo de partícula & $\begin{array}{l}\text { Densidad } \\
{\left[\mathrm{g} / \mathrm{cm}^{3}\right]}\end{array}$ & I & $\mathrm{m}$ & $\mathrm{n}$ & Epref & Fuente \\
\hline 25 & $\begin{array}{l}60 \% \text { Alumina brick (mullitebased) / Carburo de } \\
\text { silicio }\end{array}$ & 3.22 & 1.2 & 0 & 2.3 & $2.8 \mathrm{E}-4$ & Liebhard (1994) \\
\hline 26 & Sílice fundida maleable / Carburo de silicio & 3.22 & 1.2 & 0 & 2.3 & $2.8 \mathrm{E}-4$ & Liebhard (1994) \\
\hline 27 & $\begin{array}{l}\text { Ladrillo de arcilla refractaria resistente a la } \\
\text { abrasión / Carburo de silicio }\end{array}$ & 3.22 & 1.2 & 0 & 2.3 & $4 \mathrm{E}-4$ & Liebhard (1994) \\
\hline 28 & Cemento moldeable regular / Carburo de silicio & 3.22 & 1.2 & 0 & 2.3 & $4 \mathrm{E}-4$ & Liebhard (1994) \\
\hline 29 & $\begin{array}{l}\text { Super-duty Ladrillo de arcilla refractaria / Carburo } \\
\text { de silicio }\end{array}$ & 3.22 & 1.2 & 0 & 2.3 & 4.1E-4 & Liebhard (1994) \\
\hline 30 & $\begin{array}{l}\text { Thermal shock resistant castable / Carburo de } \\
\text { silicio }\end{array}$ & 3.22 & 1.2 & 0 & 2.3 & $4 \mathrm{E}-4$ & Liebhard (1994) \\
\hline 31 & Abrasion resistant castable / Carburo de silicio & 3.22 & 1.2 & 0 & 2.3 & $5 \mathrm{E}-4$ & Liebhard (1994) \\
\hline 32 & SiC cerámica sin presión / Carburo de silicio & 3.22 & 1.3 & 0.8 & 2.8 & 8.3E-5 & Wang et al. (1995) \\
\hline 33 & $\begin{array}{l}\text { SiC cerámica presada en caliente / Carburo de } \\
\text { silicio }\end{array}$ & 3.22 & 1.3 & 1.5 & 2.8 & $4-5$ & Wang et al. (1995) \\
\hline 34 & $\begin{array}{l}\text { Cerámica Isostáticamente prensada en caliente / } \\
\text { Carburo de silicio }\end{array}$ & 3.22 & 1.3 & 0.9 & 2.8 & $1.4 \mathrm{E}-5$ & Wang et al. (1995) \\
\hline 35 & $\begin{array}{l}\text { Si3N4 cerámica prensada en caliente / Carburo de } \\
\text { silicio }\end{array}$ & 3.22 & 1.3 & 0 & 4 & 4.6E-6 & Gulden (1979) \\
\hline 36 & Reaction-bonded Si3N4 ceramic / Cuarzo & 2.65 & 1.3 & 0 & 4 & 3.3E-6 & Gulden (1979) \\
\hline 37 & Si3N4 cerámica prensada en caliente / Cuarzo & 2.65 & 1 & 0 & 1 & 4.3E-7 & Gulden (1979) \\
\hline
\end{tabular}

El valor de $n$ es especifico a la velocidad de la partícula y está normalizado por $n \equiv d\left(\ln \varepsilon_{p}\right) / d\left(\ln V_{p}\right)$ y frecuentemente toma un valor de 2,3. A diferencia el exponente I es especifico al volumen de la partícula y está dado por $l \equiv d\left(\ln \varepsilon_{p}\right) / d\left(\ln v_{p}\right)$ y su valor usual es 1,2 . En cuanto a $m$ se tiene que ya se encuentra normalizado debido a que $\cos \left(\theta_{i}\right)^{m}=1$ cuando $\theta_{i}=0$, este parámetro posee valores entre 0,5 y 3,5 habitualmente. (Rosner, Tandon y Labuwsky, 1995). Mientras que la carga másica de la partícula $\omega_{p}$ en la corriente principal no sea muy pequeña, la fracción de volumen $\phi_{p}$. Corresponde a la densidad del número total de partículas $N_{p}$ y al volumen medio de las partículas $\bar{v}_{p}$.

$\phi_{p . \infty}=\rho_{\text {slurry }} \cdot \omega_{p} / \rho_{p}$

Donde $\rho_{\text {slurry }}$ es la densidad del medio corrosivo (sólido/líquido o sólido/gas), $\omega_{p}$ la carga másica de la partícula (masa partícula abrasiva/masa del fluido) y $\rho_{p}$ la densidad intrínseca de la partícula. Finalmente la tasa de erosión local puede ser calculada multiplicando la $(E R)_{c}$ por el $E_{\text {lim }}=\cos \theta^{m+1}$ y por la siguiente serie de potencias que representa el valor promedio de la tasa de erosión. (Rosner et al., 1994).

$\bar{E}_{l i m} \approx(2 / \pi)+[(11 / 16)-(8 /(3 \pi))] \cdot m+[(2 /(3 \pi))-(3 / 16)] \cdot m^{2}$

\section{METODOLOGÍA}

Con el modelo matemático descrito anteriormente se implementó un aplicativo para estimar la erosión en una pieza recubierta con un material cerámico y graficar la erosión respecto al ángulo de incidencia en el que se encuentre dicha pieza, como se describe en la figura 1. La aplicación fue realizada en SharpDevelop 4.2 el cual es un entorno de desarrollo integrado libre para lenguaje C\#. Adicionalmente el programa hace uso de las librerías de código abierto, MySql para hacer gestión de una base de datos que contiene los parámetros de erosión $\varepsilon_{p . r e f .}, l, m, n$ y ZedGraph para graficar datos en 2D.

En la figura 2 se observa la aplicación, que consta de TrackBars para que el usuario ingrese los datos de entrada, también el usuario debe escoger el tipo de recubrimiento cerámico y la partícula abrasiva que se encuentran almacenados en una base de datos por medio de un ComboBox, con estos datos el programa realiza los cálculos internamente y obtiene los datos necesarios para estimar la tasa de erosión. Los resultados se visualizan en TextBox y por medio de la gráfica de erosión local respecto al ángulo de incidencia. 


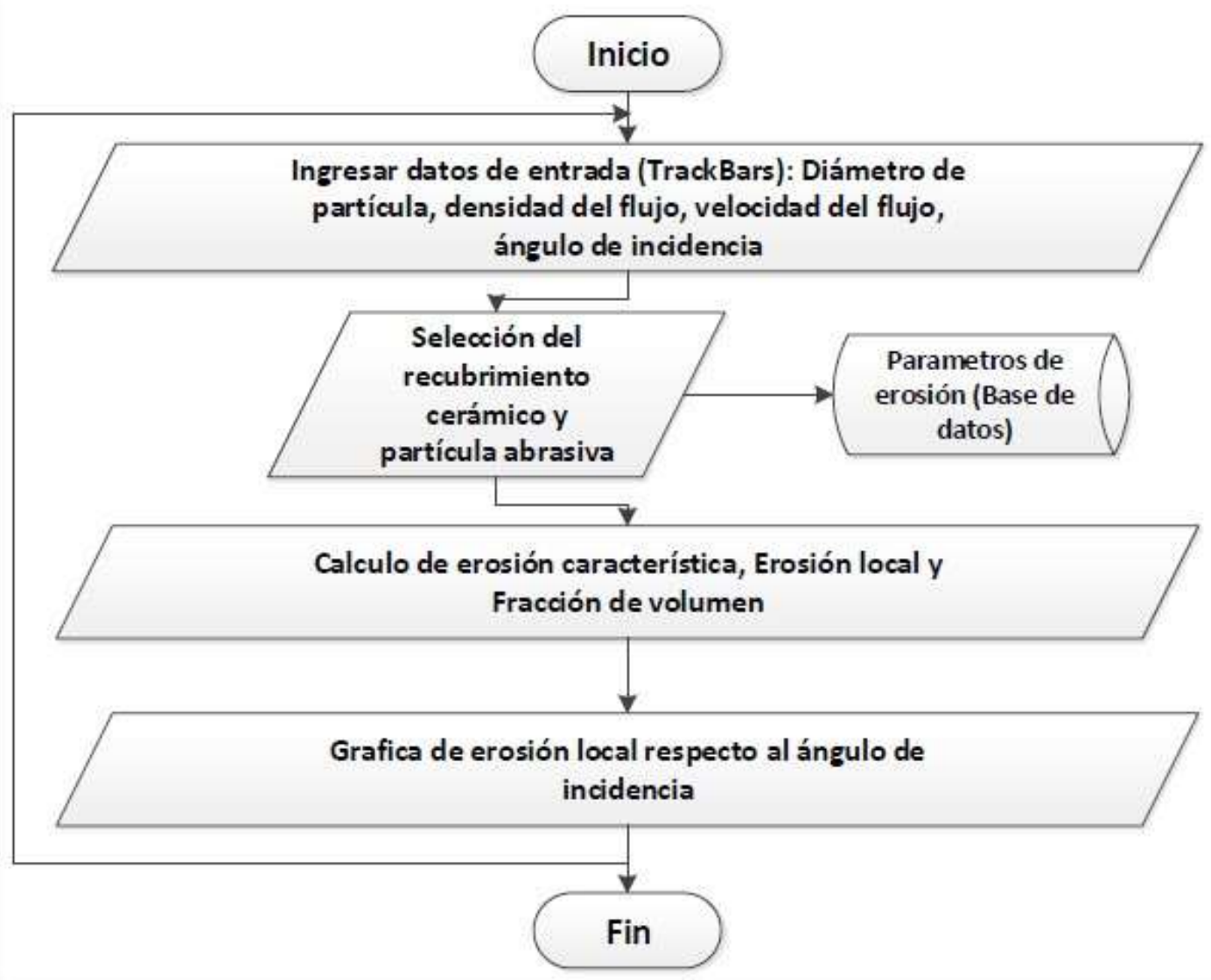

Fig. 1: Diagrama de flujo del aplicativo.

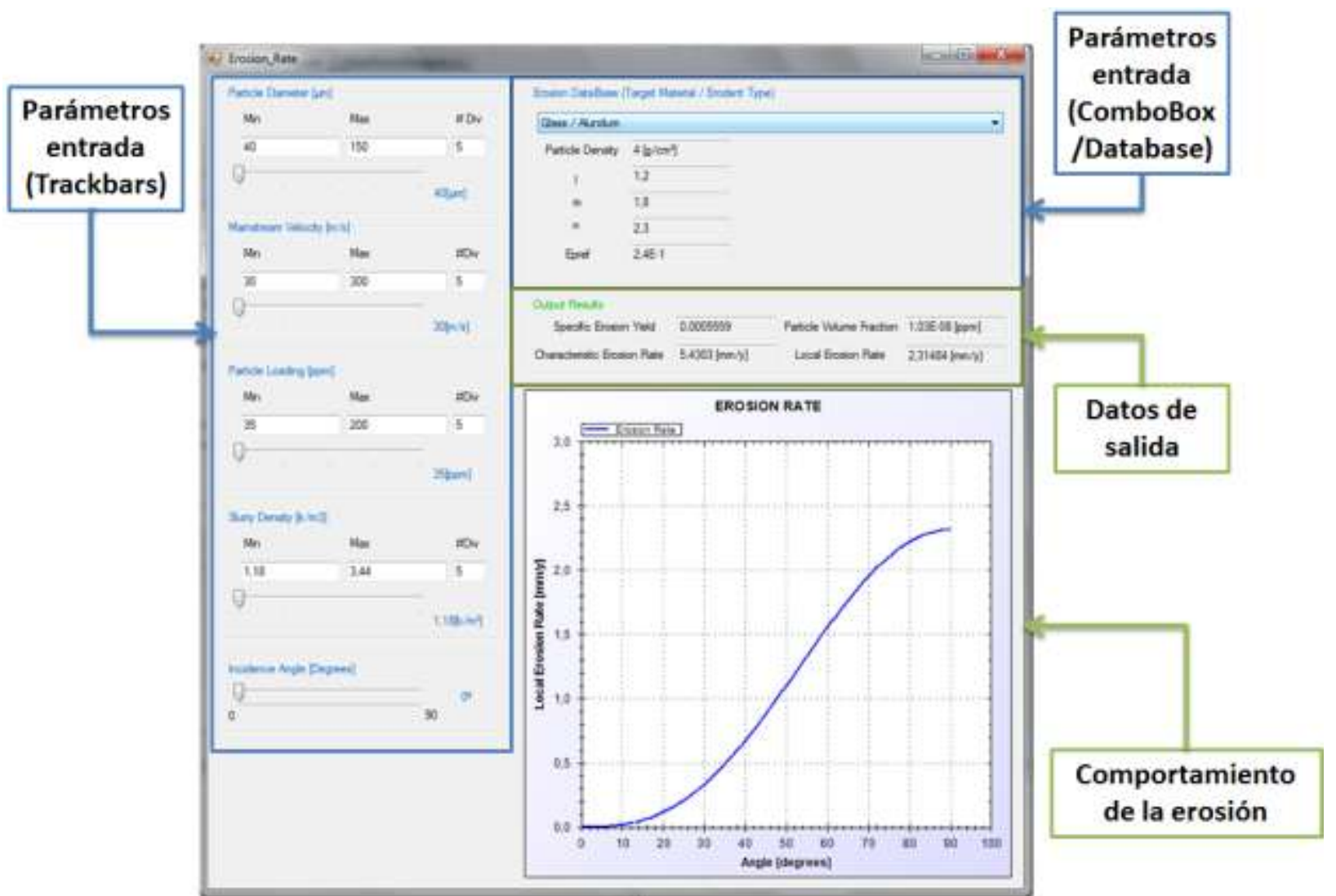

Fig. 2: Aplicación de software para la estimar el comportamiento al desgaste por erosión de recubrimientos cerámicos. 


\section{Detalle experimental}

Se depositaron películas delgadas de CrN y TiN sobre sustratos de acero AISI 4140 mediante un sistema de radio frecuencia. (13,56 MHz) magnetrón sputtering con $\operatorname{Ar}(99,9 \%$ de pureza) como gas de trabajo. Para la deposición de los recubrimientos se utilizaron blancos de 4 pulgadas de diámetro de $\mathrm{Ti}$ y $\mathrm{Cr}$ con una pureza

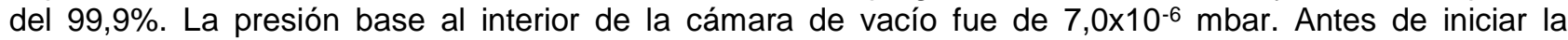
deposición los sustratos fueron sometidos a una limpieza por plasma durante 20 minutos en atmósfera de $\mathrm{Ar}$ a un bias de $-400 \mathrm{~V}$ en r.f. Durante el crecimiento, los gases de trabajo fueron una mezcla de $\operatorname{Ar}\left(93 \%\right.$ ) y $\mathrm{N}_{2}$ (7\%) con una presión total de trabajo de $6 \times 10^{-3}$ mbar a una temperatura del sustrato de $300^{\circ} \mathrm{C}$ y un bias r.f del sustrato de $-70 \mathrm{~V}$ y una potencia de $350 \mathrm{~W}$.

La evaluación del fenómeno erosivo se realizó utilizando protección catódica de $1 \mathrm{~V}$ catódico con respecto al potencial de circuito abierto del recubrimiento, de acuerdo a la norma ASTM G 119-93, e inmersos en un lodo compuesto por $3.5 \% \mathrm{NaCl}$ y $20 \%$ de partículas de $\mathrm{SiO}_{2}$, a velocidades de impacto de $11.12 \mathrm{~m} / \mathrm{s}^{2}$, impuestas por el impeler del sistema y ángulo de impacto de $90^{\circ}$. La protección catódica fue suministrada por un potenciostáto.

\section{RESULTADOS Y ANÁLISIS}

Los resultados experimentales de los ensayos de desgaste se encuentran recopilados en las tablas 2 y 3 , para poder comparar los datos prácticos con los teóricos se efectuaron conversiones a los datos experimentales para que fueran consistentes con las unidades usadas por el software. Haciendo el promedio de las diferentes tasas de erosión entregadas por la experimentación tenemos que los recubrimientos TiN y $\mathrm{CrN}$ tienen una tasa de erosión de 3,04 mm/año y 3,01 mm/año respectivamente.

Tabla 2: Datos experimentales con recubrimiento TiN.

\begin{tabular}{|c|c|c|c|c|c|}
\hline Tiempo & \multicolumn{2}{|c|}{ Desgaste } & \multicolumn{3}{|c|}{ Tasa de erosión a 90 grados } \\
\hline [minutos] & {$\left[\mathrm{g} / \mathrm{cm}^{2}\right]$} & {$\left[\mathrm{g} / \mathrm{m}^{2}\right]$} & {$\left[\mathrm{g} / \mathrm{m}^{2} / \mathrm{min}\right]$} & [g/m²/día] & [mm/año] \\
\hline 15 & $1.10 \mathrm{E}-4$ & 1.10 & 7,33E-2 & 106,00 & 4,90 \\
\hline 30 & $1.20 \mathrm{E}-4$ & 1.20 & $4,00 \mathrm{E}-2$ & 57,60 & 2,67 \\
\hline 45 & 3.30E-4 & 3.30 & 7,33E-2 & 106,00 & 4,90 \\
\hline 60 & $6.20 \mathrm{E}-4$ & 6.20 & $1,03 \mathrm{E}-1$ & 149,00 & 6,91 \\
\hline 180 & $7.10 \mathrm{E}-4$ & 7.10 & 3,94E-2 & 56,80 & 2,64 \\
\hline 540 & $8.00 \mathrm{E}-4$ & 8.00 & $1,48 \mathrm{E}-2$ & 21,30 & 9,91 \\
\hline 720 & $9.00 \mathrm{E}-4$ & 9.00 & $1,25 \mathrm{E}-2$ & 18,00 & 8,36E-01 \\
\hline 1440 & $1.00 \mathrm{E}-3$ & 10.00 & $6,94 \mathrm{E}-3$ & 10,00 & 4,64E-01 \\
\hline \multicolumn{3}{|c|}{ Promedio } & 4.55E-2 & 65.50 & 3,04 \\
\hline
\end{tabular}

Tabla 3: Datos experimentales con recubrimiento $\mathrm{CrN}$.

\begin{tabular}{|c|c|c|c|c|c|}
\hline Tiempo & \multicolumn{2}{|c|}{ Desgaste } & \multicolumn{3}{|c|}{ Tasa de erosión a 90 grados } \\
\hline [minutos] & {$\left[\mathrm{g} / \mathrm{cm}^{2}\right]$} & {$\left[\mathrm{g} / \mathrm{m}^{2}\right]$} & {$\left[\mathrm{g} / \mathrm{m}^{2} / \mathrm{min}\right]$} & [g/m²/día] & [mm/año] \\
\hline 15 & $1.10 \mathrm{E}-04$ & 1.10 & 7.33E-02 & 106,00 & 4,90 \\
\hline 30 & 2.10E-04 & 2.10 & 7.00E-02 & 101,00 & 4,68 \\
\hline 45 & $3.20 \mathrm{E}-04$ & 3.20 & 7.11E-02 & 102,00 & 4,76 \\
\hline 60 & $5.00 \mathrm{E}-04$ & 5.00 & 8.33E-02 & 120,00 & 5,57 \\
\hline 180 & $6.20 \mathrm{E}-04$ & 6.20 & $3.44 \mathrm{E}-02$ & 49,60 & 2,30 \\
\hline 540 & $6.90 \mathrm{E}-04$ & 6.90 & $1.28 \mathrm{E}-02$ & 18,40 & $8,54 \mathrm{E}-01$ \\
\hline 720 & $7.20 \mathrm{E}-04$ & 7.20 & $1.00 \mathrm{E}-02$ & 14,40 & $6,69 \mathrm{E}-01$ \\
\hline 1440 & 8.00E-04 & 8.00 & $5.56 \mathrm{E}-03$ & 8,00 & $3,72 \mathrm{E}-01$ \\
\hline \multicolumn{3}{|c|}{ Promedio } & $4.51 \mathrm{E}-02$ & 64.90 & 3.01 \\
\hline
\end{tabular}


De las pruebas experimentales se tomaron los datos consignados en la tabla 4 para las variables de entrada del software. Adicionalmente, para realizar la comparación se tuvo que actualizar la base de datos agregando los nuevos pares de recubrimiento y partícula abrasiva como se observa en la tabla 5 . Las figuras 3(a) y 3(b) muestran la respuesta entregada por el software del comportamiento de los recubrimiento frente a la erosión con respecto al ángulo de incidencia.

Tabla 4: Parámetros de entrada para el software

\begin{tabular}{|c|c|}
\hline Parámetro & Unidades \\
\hline Diámetro de la partícula & $210-300 \mu \mathrm{m}$ \\
\hline Velocidad del flujo & $11,12 \mathrm{~ms}^{-1}$ \\
\hline Carga másica de partícula & $1 \mathrm{ppm}$ \\
\hline Densidad del lodo & $21,61 \mathrm{kgm}^{-3}$ \\
\hline Ángulo de incidencia & $90^{\circ}$ \\
\hline
\end{tabular}

Tabla 5: Combinación recubrimiento cerámico partícula abrasiva.

\begin{tabular}{|c|l|c|c|c|c|c|c|}
\hline No & Material objetivo / Tipo de partícula & $\begin{array}{c}\text { Densidad } \\
{\left[\mathrm{g} / \mathrm{cm}^{3}\right]}\end{array}$ & $\mathrm{I}$ & $\mathrm{m}$ & $\mathrm{n}$ & Epref & Fuente \\
\hline 38 & Recubrimiento TiN/ Arena de Ottawa & 2,2 & 1,2 & 1,7 & 2,1 & $6,3 \mathrm{E}-3$ & Bautista et al. (2011) \\
\hline 39 & Recubrimiento CrN /Arena de Ottawa & 2,2 & 1 & 1,1 & 0,9 & $4,8 \mathrm{E}-3$ & Barshilia et al. (2007) \\
\hline
\end{tabular}

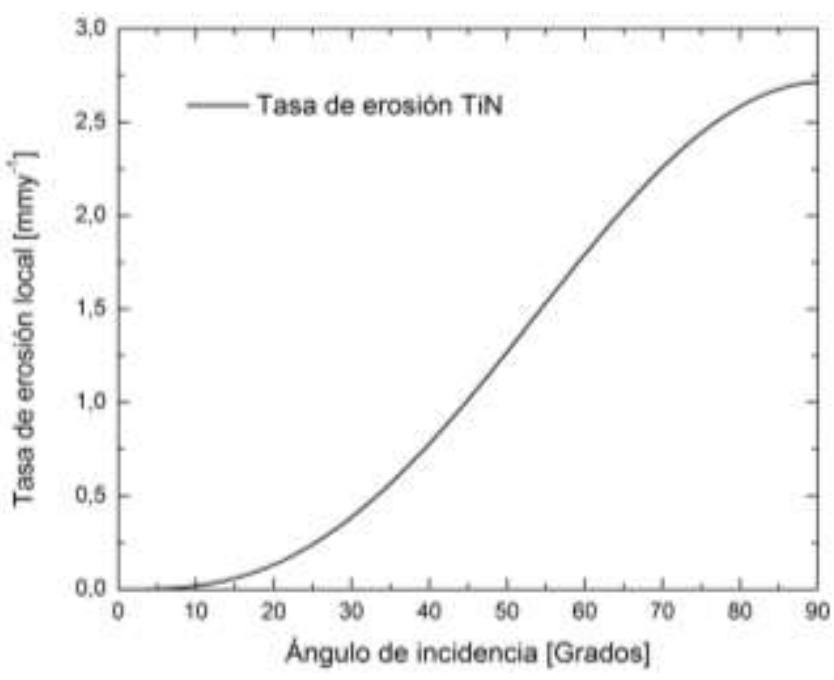

(a)

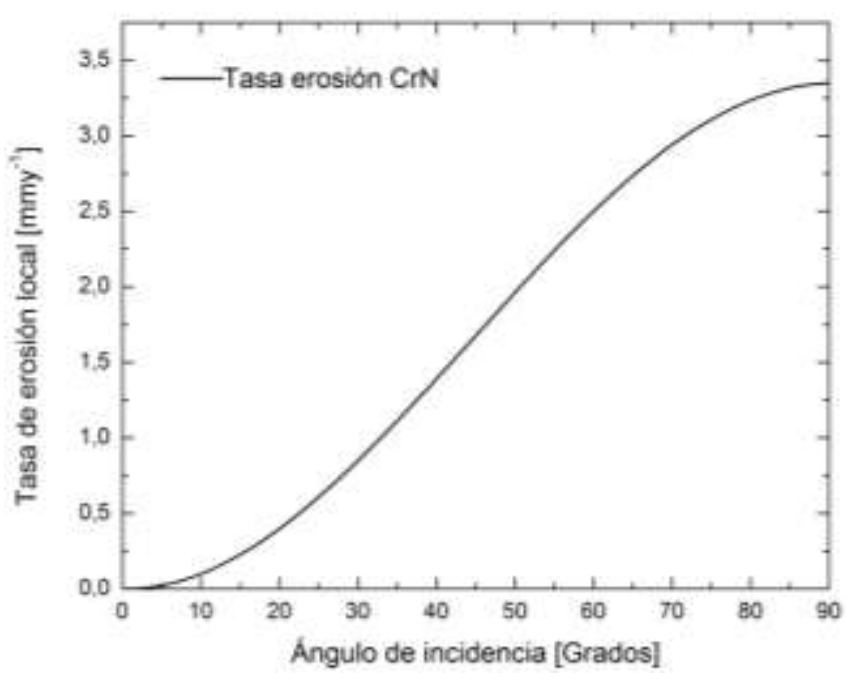

(b)

Fig. 3: Tasa de erosión de $\mathrm{TiN}(\mathrm{a})$ y $\mathrm{CrN}(\mathrm{b})$ entregada por el software.

La tasa de erosión adquiere su valor máximo cuando el ángulo de incidencia es $90^{\circ}$ para el caso del recubrimiento TiN se estima en 2,73 mm/año y para el CrN 3,36 mm/año. La tabla 6 muestra la comparación de las medidas experimentales con las medidas estimadas mediante el aplicativo de software, donde se evidencia un error de $11,36 \%$ en el caso del recubrimiento de TiN y $10,47 \%$ en el recubrimiento CrN, lo cual permite usar el software como una herramienta para estimar el desgate erosivo, evitando la realización de ensayos experimentales para el estudio de diferentes recubrimientos.

Tabla 6: Contraste entre datos teóricos con prácticos.

\begin{tabular}{|c|c|c|c|}
\hline Recubrimiento & Experimental & Software & Error \\
\hline TiN & $3,04 \mathrm{~mm} / \mathrm{año}$ & $2,73 \mathrm{~mm} / \mathrm{año}$ & $11,36 \%$ \\
\hline $\mathrm{CrN}$ & $3,01 \mathrm{~mm} / \mathrm{año}$ & $3,36 \mathrm{~mm} / \mathrm{año}$ & $10,47 \%$ \\
\hline
\end{tabular}




\section{CONCLUSIONES}

Mediante el aplicativo desarrollado se simplificó el cálculo para predecir la tasa de erosión de un recubierto cerámico expuesto a un flujo de partículas abrasivas, describiendo como el proceso de erosión es afectado por el tamaño, la velocidad de incidencia y ángulo de incidencia de la partícula abrasiva mediante los parámetros de erosión I, n, $\mathrm{m}$ almacenados en la base de datos.

El aplicativo desarrollado permite estimar el comportamiento de un material cerámico frente a la erosión debida al impacto de partículas abrasivas, variando de manera sencilla diferentes parámetros como: el diámetro y densidad de la partícula abrasiva, la velocidad y el ángulo de incidencia del flujo, la densidad del lodo, la carga másica de partículas y los parámetros de erosión I, m, n, $\varepsilon_{\text {pref }}$, lo cual simplifica el estudio, ya que es complejo recrear los diferentes ambientes experimentalmente.

\section{AGRADECIMIENTOS}

Los autores agradecen al Programa Jóvenes Investigadores de COLCIENCIAS. Además, a la Vicerrectoría de Investigaciones de la Universidad Militar Nueva Granada por la financiación de este trabajo a través del proyecto ING 1767 (2015).

\section{REFERENCIAS}

Aperador, W., C. Amaya y C. España, Resistencia a la corrosión de las multicapas de [TiN/AlTiN]n Depositadas sobre acero al carbono AISI 1045, Revista Dyna, 78(165), 183-189 (2011).

Aperador, W., A.S. Mejía y J. Caicedo, Comportamiento de Corrosión-Erosión en Recubrimientos de TiN/ZrN Depositados sobre Acero AISI 4140, doi: 10.4067/S0718-07642014000100004, Inf. Tecnol. (en línea), 25(1), 23-32 (2014)

Aperador, W., y otros autores. Bilayer period effect on corrosion-erosion resistance for [TiN/AlTiN]n multilayered growth on AISI 1045 steel, Journal of Physics and Chemistry of Solids, 71(12), 1754-1759 (2010).

Aperador, W., C. Ramírez y J. Bautista, Sinergia entre la corrosión erosión del acero 1045 recubierto por multicapas de TiN/TiAIN, Revista Mexicana De Física, 57(4), 350-355 (2011)a.

Barshilia, C., y otros autores. Growth and characterization of TiAIN/CrAIN superlattices prepared by reactive direct current magnetron sputtering. Journal of Vacuum Science \& Technology A, 27(1), 29-36 (2009).

Barshilia, C., y otros autores. Nanolayered multilayer coatings of CrN/CrAIN prepared by reactive DC magnetron sputtering. Applied Surface Science, 253(11), 5076-5083 (2007).

Bautista, J., W. Aperador y C. España, Desgaste abrasivo y corrosivo de recubrimientos TiN/TiAlN y TiN/AITiN, Editorial Académica Española, (2011).

Bitter, J.G.A. A study of erosion phenomena, Parts I and II, Wear, 6(1-3), 5-21 y 169-190 (1963).

Caicedo, J.C., y otros autores. Improvement of the electrochemical behavior of AISI 4140 steel substrate using [TiCN/TiNbCN]n multilayers system, Niobium: Properties, Production and Applications, (2011).

Caicedo, J.C., y otros autores. Effect of applied bias voltage on corrosion-resistance for TiC1-xNx and Ti1-xNbxC1-yNy coatings. Applied Surface Science, 256(9), 2876-2883 (2010).

Caicedo, J. C., y otros autores. Corrosion-Erosion Effect on TiN/TiAIN Multilayers, Journal of materials engineering and performance. 21(9) 1949-1955 (2012).

Chu, Xi. y S. Barnett, Model of superlattice yield stress and hardness enhancements, Journal of Applied Physics, 77(9) , $4403-4412$ (1995).

Crowley, M.S. Influence of particle size on erosion resistance of refractory concretes, Ceramic Bulletin, 48(7), 707-710 (1969).

Finnie, I. Erosion of surfaces by solid particles, Wear, 3, 87-103 (1960).

Gee, M.G., y otros autores, Wear of Hardmetals, Comprehensive Hard Materials, Elsevier, Volume 1: Hardmetals, pp 363-383, (2014). 
Gulden, M.E. Solid-particle erosion of high-technology ceramics $\left(\mathrm{Si}_{3} \mathrm{~N}_{4}\right.$, glass-bonded $\mathrm{Al}_{2} \mathrm{O}_{3}$, and $\left.\mathrm{MgF}_{2}\right)$, En: W.F. Adler (ed.), Erosion: Prevention and Useful Applications, ASTM Special Technical Publication 664, (1979).

Head W.J. y M.E. Harr, The development of a model to predict the erosion of materials by natural contaminants. Wear, 15(15), 1-46 (1970).

Holmberg, K., A. Matthews y H. Ronkainen, Coatings Tribology- contact mechanisms and surface design, Tribology International, 31(1-3), 107-120 (1998).

Jehn, H.A. Improvement of the corrosion resistance of PVD hard coating-substrate systems, Surface and Coatings Technology, 125(1-3), 212-217 (2000).

Junhua, Xu., Li. Geyang y Gu. Mingyuan, The microstructure and mechanical properties of TaN/TiN and TaWN/TiN superlattice films. Thin Solid Films, 370(1-2), 45-49 (2000).

Keairns, D.L., W.C. Yang y W.G. Vaux, Design of refractories for coal gasification and combustion systems, EPRI AF-1151, Electric Power Research Institute, Pale Alto, CA, (1979).

Khalil, Y.F. y D.E. Rosner, Erosion rate prediction and correlation technique for ceramic surfaces exposed to high speed flows of abrasive suspensions. Wear, 201(1-2), 64-79 (1996).

Liebhard, M.K. Erosion and corrosion of refractories in circulating-fluidized-bed combustors, EPRI TR104039, Electric Power Research Institute, Pale Alto, CA, (1994).

Nieto, J., y otros autores. Evaluación de la influencia del voltaje bias sobre la resistencia a la corrosión de películas delgadas de AINbN, Revista Dyna, 77(162), 161-168 (2009).

Rosner, D.E., y otros autores. Prediction/correlation of particle deposition rates from polydispersed flowing suspensions and the nature/properties of resulting deposits, Proc. Ist Int. Particle Technology Forum, AIChE, Denver, CO, 374-381 (1994).

Rosner, D.E., P. Tandon y M.J. Labuwsky, Rapid estimation of cylinder erosion rates in abrasive dust-laden streams. AIChE JOURNAL, 41(5), 1081-1098 (1995).

Wang, D.F., J.H. She y Z.Y Ma, Effect of microstructure on erosive wear behavior of SiC ceramics, Wear, 180(1-2), 35-41 (1995).

Wiederhorn, S.M. y D.E. Roberts, A technique to investigate high temperature erosion of refractories, Ceramic Bulletin, 55(2), 185-189 (1976).

Yashar, P. y W. Sproul, Nanometer scale multilayered hard coatings. Vacuum, 55(3), 179-190 (1999). 
